\title{
Effect of exercise and high carbohydrates diet in the T-lymphocytes of Peyer's patches of Balb/c mice
}

\author{
B. E. Martínez-Carrillo ${ }^{1,2}$, M. G. Zúñiga-Torres ${ }^{1}$, R. A. Jarillo-Luna ${ }^{2}$, R. Valdés-Ramos ${ }^{1}$, \\ V. Rivera-Aguilar ${ }^{2}$, R. Campos-Rodriguez ${ }^{2}$, R. V. Pardo-Morales ${ }^{1}$ and C. Rosales-Gómez ${ }^{2}$ \\ ${ }^{1}$ Center for Research and Graduate Studies in Health Sciences, Faculty of Medicine, Universidad Autónoma del Estado \\ de México and ${ }^{2}$ Laboratorio de Morfología, Escuela Superior de Medicina, Instituto Politécnico Nacional, México
}

It has been shown that exercise induces considerable changes in the body, particularly in the mucosal immune system ${ }^{(1)}$; the current lifestyles have promoted an increase in the consumption of high carbohydrate diets ( $\uparrow \mathrm{CHO})$; these two factors result in particular effects on Peyer's patches (PP) ${ }^{(2)}$. The objective of this study was to evaluate the effect of moderate exercise and $\uparrow$ CHO on PP's T-cells (TLc) in $\mathrm{Balb} / \mathrm{c}$ young mice. Thirty-two 21-d-old male mice were divided into four groups, two groups were taught to swim for $30 \mathrm{~min} 5 \mathrm{~d}$ per week during 9 weeks; one of which was fed a commercial control diet (CONTROL/E) and the other was fed a high carbohydrate diet ( $\uparrow$ CHO/E) (DIO Rodent Purified Diet, Cat. 58Y2; energy: $4.65 \mathrm{kcal} / \mathrm{g}(19.4556 \mathrm{~kJ} / \mathrm{g}$ ), the other two groups were fed the same diets but were not made to swim (CONTROL and $\uparrow \mathrm{CHO}$ ). Small intestines were dissected, PP were cut, macerated, filtered and centrifuged to obtain the lymphocyte pellet; cells were stained with anti-CD3+, CD4 + and CD8 + antibodies and analysed by flow cytometry (Facs Diva, BD $\left.{ }^{\circledR}\right)$. The experimental diet increased the weight of mice who exercised (23.5, SD 1.3) compared with the non-exercise group (19.4, SD 1.8) and the control group (20.2, SD 1.4), $P \leq 0.005$. CD3 + TLc were decreased in the $\uparrow$ CHO/E group (31.1, SD 1.0) compared with the CONTROL/E group (38.8, SD 2.5), T 8.103, $P \leq 0.001$; CD3 +/CD4 + (23.2, SD 0.2), CD3 +/CD8 + (3.5 \pm 0.5$)$ also decreased compared with CONTROL/E group (33.5, sD 1.97 and 4.9, sD 0.5; t 14.8 and t 6.014 respectively, $P \leq 0.001$ ). The combination of exercise and $\uparrow \mathrm{CHO}$ decreased significantly PP TLc, we can conclude that the intake of a high carbohydrate diet together with exercise attenuates lymphocytes transit, as well as increasing the weight of the mice (Fig. 1).

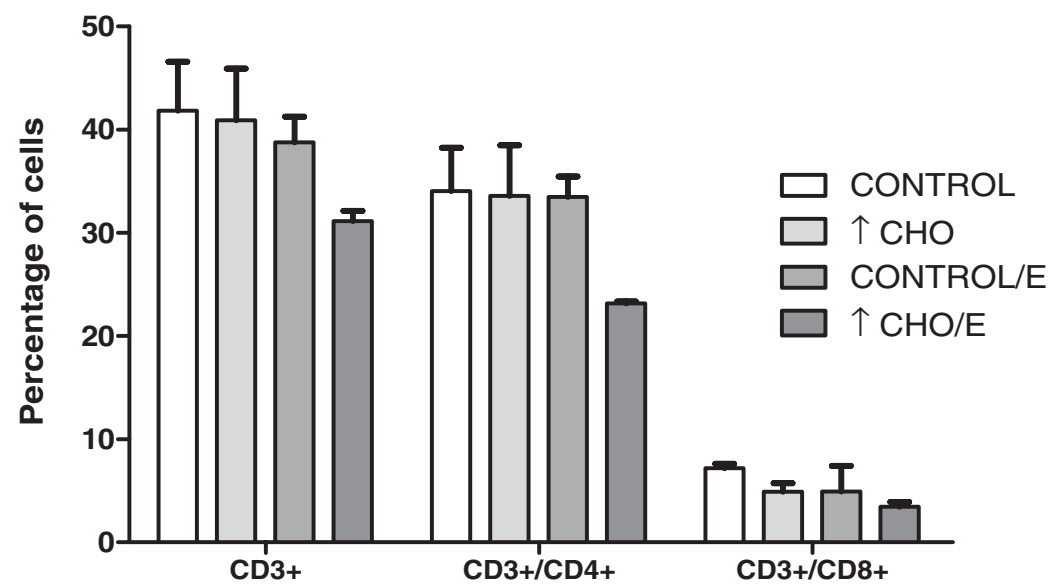

Fig. 1. Percentage of lymphocytes CD3 +, CD3 +/CD4 + and CD3 +/CD8 + from PP from Balb/c mice, feeding with standard diet (CONTROL) or high carbohydrate diet ( $\uparrow \mathrm{CHO}$ ); with or without $30 \mathrm{~min}$ of exercise (/E). Values are show in means and standard deviations (SD) $n 8$, differences were statistically significant by Student's $t$ test at $P \leq 0.001$. CONTROL: group with standard diet without exercise; $\uparrow \mathrm{CHO}$ : group with high carbohydrate diet; CONTROL/E: group with standard diet with exercise; $\uparrow \mathrm{CHO} / \mathrm{E}$ : group feeding with high carbohydrate diet, and with exercise.

This project was financed by Universidad Autónoma del Estado de México.

1. Pedersen BK \& Hoffman-Goetz L (2000) Physiol Rev 80, 1055-1081.

2. Rogers CJ, Berrigan D, Zaharoff DA et al. (2008) J Nutr 138, 115-122. 